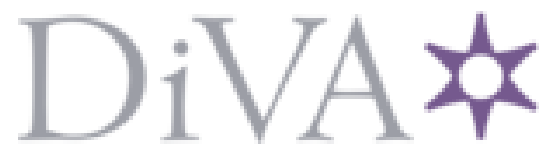

http://www.diva-portal.org

Postprint

This is the accepted version of a paper presented at 18th European Control Conference (ECC), Naples, ITALY, JUN 25-28, 2019.

Citation for the original published paper:

Čičić, M., Johansson, K H. (2019)

Energy-optimal platoon catch-up in moving bottleneck framework

In: 2019 18TH EUROPEAN CONTROL CONFERENCE (ECC) (pp. 3674-3679). IEEE https://doi.org/10.23919/ECC.2019.8795754

N.B. When citing this work, cite the original published paper.

Permanent link to this version:

http://urn.kb.se/resolve?urn=urn:nbn:se:kth:diva-263384 


\title{
Energy-optimal platoon catch-up in moving bottleneck framework
}

\author{
Mladen Čičić ${ }^{1}$ and Karl Henrik Johansson ${ }^{1}$
}

\begin{abstract}
Truck platooning, where vehicles drive close together in order to reduce air drag, is a promising technology that is slowly reaching maturity. In order to fully exploit its potential, we will need to be able to efficiently form platoons enroute, while driving on the road, by having the vehicles adjust their speeds so that they catch-up and merge into a platoon. Since this means that the participating vehicles will have to deviate from their own optimal speed profiles, experiencing unpredicted disturbances caused by the surrounding traffic might lead to higher fuel consumption. Therefore, in order to increase the fuel savings and improve their predictability, we devise a control strategy that takes into account the traffic conditions and yields optimal catch-up speeds for both the leader and the follower vehicle. The control strategy is based on minimizing total energy consumption, and it models the vehicles as moving bottlenecks. We compare this strategy to the one that does not consider the influence of traffic, and show that it achieves better results in terms of energy consumption savings.
\end{abstract}

\section{INTRODUCTION}

Recent years have seen an accelerated push towards heavyduty vehicle platooning $[1,2]$. Traditionally, such platooning was primarily regarded as means of reducing the air drag acting on the vehicles [3], and thus fuel consumption, but there are also other benefits, like facilitating a higher level of automation. There has been much work done on controlling the vehicles inside a platoon [4], [5], and this technology is slowly transitioning from academia to industry. There are other aspects of platooning that still require more research, including how truck platoons influence traffic, how platoons should be formed, and how to make decisions on which vehicles should platoon with which other vehicles [6].

Real-time platoon formation, where vehicles attempt to form platoons en-route, is one of these open problems. Dynamic planning strategies have been proposed [7], with the platooning coordinator selecting vehicles to platoon and sending them jointly fuel-optimal speed profiles and routes, following which the vehicles catch up, merge into a platoon, drive together for some time, and then diverge. However, this also means that the participating vehicles will have to deviate from their own optimal speed profiles, which entails higher

\footnotetext{
*The research leading to these results has received funding from the European Union's Horizon 2020 research and innovation programme under the Marie Skłodowska-Curie grant agreement No 674875, VINNOVA within the FFI program under contract 2014-06200, the Swedish Research Council, the Swedish Foundation for Strategic Research and Knut and Alice Wallenberg Foundation. The authors are affiliated with the Wallenberg AI, Autonomous Systems and Software Program (WASP).

${ }^{1}$ Department of Automatic Control, KTH Royal Institute of Technology, Stockholm, Sweden Mladen Čičić: cicic@kth.se, Karl Henrik Johansson: kallej@kth.se
}

fuel consumption during the catch-up and merging phase that we hope to offset by fuel savings during the platooning phase [8]. If the platoon merging fails or is delayed due to some unpredicted disturbance [9], the net energy consumption could be higher than expected, potentially leading to more fuel being spent compared to the case when the vehicles would continue driving at their individual optimal speeds. It is therefore important to have a good prediction of when the platoon merging will be completed, so as to be able to calculate predicted energy savings and make a better informed decision on whether to attempt to form a platoon at all. This problem was studied in [10] and [11], as well as in [12]. In these papers the authors did consider the influence of traffic, but did not study how to compensate for it. We identified two potential causes for deviating from nominal platoon merging times [13], the influence of road grade and the influence of the surrounding traffic, and addressed the first one.

The aim of this paper is to address the energy-optimal platoon catch-up, taking the surrounding traffic into account. In Section II we formulate the problem and the cost function we want to minimize. In Section III, we further build upon an extension of the cell transmission model (CTM) [14] with moving bottlenecks, presented in [15]. This addition enables modelling long moving bottlenecks, and allows situations with multiple moving bottlenecks in the same cell, and moving bottleneck merging. Next, in Section IV we use this framework to model the interaction between the heavy-duty vehicles and the surrounding traffic. We formulate a traffic condition dependent cost function based on accurate prediction of energy expenditure until the end of the platooning section. Minimizing this cost function, we calculate energyoptimal platoon catch-up speed pairs, taking the influence of traffic into account, which are applied as control input to the vehicles. Finally, in Section V we test this control in simulations and in Section VI conclude.

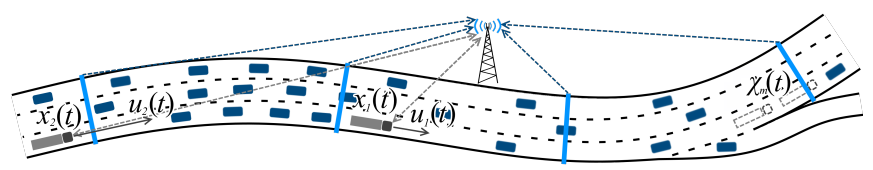

Fig. 1: Platoon catch-up assisted by a controller in the infrastructure. Traffic and other road data is collected and, together with vehicle starting positions $x_{1}(t)$ and $x_{2}(t)$, used to calculate optimal catch-up speeds $u_{1}(t)$ and $u_{2}(t)$, and platoon merging position prediction $\chi_{m}(t)$. 


\section{Problem Formulation}

Most proposed large-scale platooning solutions involve a layered control architecture [1]. At the higher layer, platooning coordinator plans the transport assignments and optimizes vehicle routes, including identifying and managing potential platoons. At the middle layer, vehicles receive their routes and generate their speed profiles, which the lower layer control is tasked to follow. Platoon catch-up coordination is handled at the middle layer.

Assume a potential platooning pair, driving along the common stretch of road, has been identified (see Fig. 1). We adopt the coordinate system so that at $\tau=0$, the leading vehicle's position is $\chi_{1}(0)=\chi_{0}$, and the following vehicle's position $\chi_{2}(0)=0$. Vehicles' motion along the road can be described by

$$
\dot{\chi}_{i}=v_{i}(\tau), \quad \tau \leq \tau_{m}
$$

where $i=1,2$, and the dot denotes differentiation by $\tau$,

$$
\tau_{m}=\min \left(\tau \in \mathbb{R}_{\geq 0} \mid \chi_{1}(\tau)-\chi_{2}(\tau) \leq d_{p}\right)
$$

is the time when the vehicles merge into a platoon, $d_{p} \approx 0$ is some predefined maximum distance between two vehicles we consider to be in a platoon, and

$$
v_{i}(\tau)=\min \left(u_{i}(\tau), v_{\max }\left(\chi_{i}(\tau), \tau\right)\right),
$$

where $v_{\max }(\chi, \tau)$ is the maximum speed at position $\chi$ at time $\tau$ imposed by some external influences. That is, vehicles attempt to drive at constant reference speeds $u_{1}$ and $u_{2}$, respectively, until they have successfully merged into a platoon. The platoon will then proceed to drive at speed $v_{p}=u_{p}$, calculated so that it reaches the end of their shared route segment $\chi_{f}$ at some specified time $\tau_{f}$.

Although in reality, vehicles might have some degree of flexibility with regard to timing, by only considering one $\tau_{f}$, we ensure that the comparison between different pairs of speeds $\left(u_{1}, u_{2}\right)$ is fair. Otherwise, the delay would have to be reflected in the cost function and the optimization problem will become more complicated. Instead, in order to negate the ostensible energy savings by delaying the vehicle, we will assume that both vehicles need to be at position $\chi_{f}$ at time $\tau_{f}$ regardless of whether or not they platoon, and take $\tau_{f}$ so that it satisfies the most stringent constraints the two vehicles have.

We focus on reducing the total work required to overcome the resistive forces acting on the vehicles. The three major external forces acting on vehicles are air drag, rolling resistance and gravity. We assume that road grade is zero, allowing us to focus solely on reducing air drag, since the contribution of rolling resistance will be the same whether or not the vehicles adjust their speeds and attempt to merge into a platoon.

Air drag acting on a vehicle can be modelled as

$$
F_{a d}(v, d)=k_{a} \phi_{a}(d) v^{2},
$$

where $k_{a}$ is a constant that depends on the air density, the cross-section area of the vehicle, and the air drag coefficient, $v$ is the speed of the vehicle, and $\phi_{a}(d) \in[0,1]$ is the portion of air drag that acts on a platooning vehicle, with $d$ being the inter-vehicular distance. Assuming $d$ is constant yields the resistive force acting on the platoon leader $F_{a d, l}(v)=k_{a} v^{2}$ and on followers $F_{a d, f}(v)=k_{a} v^{2} \phi_{f}, \phi_{f}=\phi_{a}(d)<1$.

The cost function related to the air drag component of the overall resistive force can therefore be written

$$
J(v)=\int_{0}^{\tau_{m}}\left[v_{1}^{3}(\tau)+v_{2}^{3}(\tau)\right] d \tau+\phi \int_{\tau_{m}}^{\tau_{f}} v_{p}^{3}(\tau) d \tau .
$$

In addition, to obey the timing and platoon merge constraints, we require that

$$
\begin{gathered}
\int_{0}^{\tau_{m}}\left[v_{2}(\tau)-v_{1}(\tau)\right] d \tau=\chi_{0}, \\
\int_{0}^{\tau_{m}} v_{1}(\tau) d \tau+\int_{\tau_{m}}^{\tau_{f}} v_{p}(\tau) d \tau=\chi_{f}-\chi_{0}, \\
\int_{0}^{\tau_{m}} v_{2}(\tau) d \tau+\int_{\tau_{m}}^{\tau_{f}} v_{p}(\tau) d \tau=\chi_{f} .
\end{gathered}
$$

We also need to constrain the reference vehicle speeds to lie within some range, $u_{\min } \leq u_{1} \leq u_{\max }, u_{\min } \leq u_{2} \leq u_{\max }$, which will lead to similar constraints for the actual vehicle speeds $v_{1}$ and $v_{2}$.

By $\phi$ we denote the total air drag coefficient of the platoon, which is the sum of air drag coefficients of its individual vehicles. Assuming there are two vehicles in a platoon driving with intervehicular distance of about $20 \mathrm{~m}$, we typically have $\phi \approx 1.7$ [3].

Ideally, the metric that we would like to use to evaluate the optimality of chosen catch-up speeds would be fuel consumption. However, getting accurate fuel consumption figures can be very difficult, and will depend on the properties of the vehicles in question. Instead, by using the required energy, we obtain a more general result.

If we assume that the vehicles follow their reference speeds perfectly, $v_{i}(\tau)=u_{i}, v_{p}(\tau)=u_{p}$, we have $\tau_{m_{0}}=\frac{\chi_{0}}{u_{2}-u_{1}}$ and $u_{p}=\frac{\chi_{f}\left(u_{2}-u_{1}\right)-\chi_{0} u_{2}}{\tau_{f}\left(u_{2}-u_{1}\right)-\chi_{0}}$. We can then simplify (1) and combine it with conditions (2) to get

$$
\begin{aligned}
J_{0}(u) & =\left(u_{1}^{3}+u_{2}^{3}\right) \tau_{m_{0}}+\phi \frac{\left(\chi_{f}-\chi_{m_{0}}\right)^{3}}{\left(\tau_{f}-\tau_{m_{0}}\right)^{2}} \\
& =\frac{\left(u_{1}^{3}+u_{2}^{3}\right) \chi_{0}}{u_{2}-u_{1}}+\phi \frac{\left(\chi_{f}\left(u_{2}-u_{1}\right)-u_{2} \chi_{0}\right)^{3}}{\left(u_{2}-u_{1}\right)\left(\tau_{f}\left(u_{2}-u_{1}\right)-\chi_{0}\right)^{2}} .
\end{aligned}
$$

This cost function is parametrized by $\chi_{0}, \chi_{f}, \tau_{f}$ and $\phi$, and we seek to minimize it by choice of $u_{1}$ and $u_{2}$.

Denote by $u_{10}=\frac{\chi_{f}-\chi_{0}}{\tau_{f}}$ and $u_{20}=\frac{\chi_{f}}{\tau_{f}}$ the constant speeds individual vehicles would need to keep in order to reach $\chi_{f}$ at $\tau_{f}$, and assume this is possible without violating the constraints on minimum and maximum speed. Note that, although possible, it will never be beneficial for the leader to go faster than $u_{10} \leq u_{\max }$ nor for the follower to go slower than $u_{20} \geq u_{\min }$. Therefore, we can further tighten the constraints to $u_{\min } \leq u_{1} \leq u_{10}$ and $u_{20} \leq u_{2} \leq u_{\max }$.

The minimization problem that we solve to calculate optimal $u_{1}$ and $u_{2}$ then becomes

$$
\begin{array}{ll}
\underset{u_{1}, u_{2}}{\operatorname{minimize}} & J_{0}(u) \\
\text { subject to } & u_{\min } \leq u_{1} \leq u_{10} \\
& u_{20} \leq u_{2} \leq u_{\max } .
\end{array}
$$

This is a convex problem that can easily be solved numerically. By solving this problem, we can calculate energyoptimal platoon catch-up speeds when not taking traffic 
conditions into account, i.e., the speeds that would be energyoptimal if the vehicles would not be affected by traffic.

However, since we assumed no interference from traffic and other extraneous factors that might render it impossible for the follower vehicle to maintain its optimal speed, we might see discrepancies in behaviour that will make this solution suboptimal. Consequently, the energy savings will be lower than expected, further motivating including the traffic conditions in the optimization problem.

To summarize, we are looking to calculate the optimal catch-up speeds for two vehicles attempting to form a platoon in traffic. The cost function that we minimize in order to do this is given in equation (1), and it reflects the total work the vehicles need to do in order to overcome the air resistance during the whole process of catching up, forming a platoon and driving together until the end of the common road segment, taking the influence of traffic into account. Next section gives the required model, which captures the interaction between the vehicles in question and the rest of the traffic. This model is then used in the following section to solve this problem.

\section{TRAFFIC MODEL WITH MOVING BOTTLENECKS}

In this section we develop a traffic model with two moving bottlenecks, where the bottlenecks represent the two trucks forming a platoon in traffic. The traffic model used here is an extension of CTM with moving bottlenecks from [15]. The extension will be in two ways: we drop the assumption that the length of the moving bottleneck is negligible compared to cell lengths and introduce a way of handling multiple moving bottlenecks in adjacent cells, as well as handling their possible merging.

Following the notation from [15], we denote the traffic density in cell $i$ at time $t$ by $\rho_{i}(t)$, and the flow from cell $i$ to $i+1$ by $q_{i}(t)$. Parameters $V, W, \sigma$ and $P$ denote the free flow speed, congestion wave speed, critical density and jam density, respectively, and we write the flux function $Q(\rho)$. Parameters and variables for the moving bottleneck zone are indicated by subscript $b$, and we denote the speed of the moving bottleneck by $u_{b}$.

First, we define functions

$$
\begin{aligned}
r_{f}\left(\rho_{b}, u_{b}\right) & =\frac{Q_{b}\left(\rho_{b}\right)-u_{b} \rho_{b}}{V-u_{b}}, \\
r_{c}\left(\rho_{b}, u_{b}\right) & =\frac{W P-Q_{b}\left(\rho_{b}\right)+u_{b} \rho_{b}}{W+u_{b}}, \\
r_{f}^{-1}\left(\rho, u_{b}\right) & =\frac{Q(\rho)-u_{b} \rho}{V_{b}-u_{b}}, \\
r_{c}^{-1}\left(\rho_{b}, u_{b}\right) & =\frac{W_{b} P_{b}-Q(\rho)+u_{b} \rho}{W_{b}+u_{b}},
\end{aligned}
$$

which give us intersections between the flux function $Q(\rho) \quad\left(Q_{b}(\rho)\right)$ and a line with slope $u_{b}$ originating from $\left(\rho_{b}, Q_{b}\left(\rho_{b}\right)\right)((\rho, Q(\rho)))$, respectively, see Fig. 2. It is easy to check that $r_{f, c}^{-1}\left(r_{f, c}\left(\rho, u_{b}\right), u_{b}\right)=\rho$ and $r_{f, c}\left(r_{f, c}^{-1}\left(\rho, u_{b}\right), u_{b}\right)=\rho$.

The Riemann problems [16] for the moving bottleneck

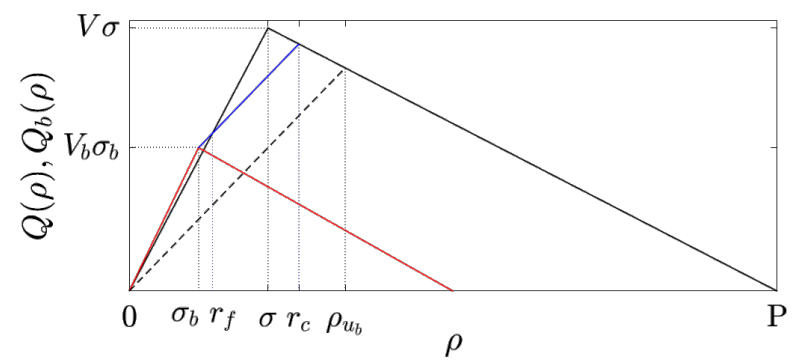

Fig. 2: Default and reduced flux function $Q(\rho)$ (black) and $Q_{b}(\rho)$ (red).

boundaries can be written

$$
\begin{gathered}
\partial_{\tau} \rho+\partial_{x}\left(Q_{ \pm}(\rho, x, \tau)\right)=0, \\
Q_{ \pm}\left(\rho, u_{b}, x, \tau\right)= \begin{cases}Q_{-}(\rho), & x<u_{b} \tau, \\
Q_{+}(\rho), & x>u_{b} \tau,\end{cases} \\
\rho(x, 0)= \begin{cases}\rho_{-}, & x<0, \\
\rho_{+}, & x>0 .\end{cases}
\end{gathered}
$$

We will consider two types of Riemann problems, for the moving bottleneck head (its downstream end) and for the moving bottleneck tail (its upstream end).

Consider first the Riemann problem for the moving bottleneck head. In this case, we have $Q_{-}(\rho)=Q(\rho)$, $Q_{+}(\rho)=Q_{b}(\rho)$ and $\rho_{-}=\rho_{b}$. Depending on $\rho_{b}$ and $\rho_{+}$, the entropy solution is:

- If $\rho_{b} \leq \sigma_{b}$ and $\rho_{+}<r_{c}\left(\rho_{b}, u_{b}\right)$ :

$$
\rho(x, \tau)= \begin{cases}\rho_{b}, & x<u_{b} \tau, \\ r_{f}\left(\rho_{b}, u_{b}\right), & u_{b}<x<\Lambda\left(r_{f}\left(\rho_{b}, u_{b}\right), \rho_{+}\right) \tau, \\ \rho_{+}, & x>\Lambda\left(r_{f}\left(\rho_{b}, u_{b}\right), \rho_{+}\right) \tau .\end{cases}
$$

- If $\rho_{b}<r_{f}^{-1}\left(\rho_{+}, u_{b+}\right)$ and $\rho_{+}>r_{c}\left(\sigma_{b}, u_{b+}\right)$ :

$$
\rho(x, \tau)= \begin{cases}\rho_{b}, & x<\Lambda_{b}\left(\rho_{b}, r_{c}^{-1}\left(\rho_{+}, u_{b+}\right)\right) \tau, \\ r_{c}^{-1}\left(\rho_{+}, u_{b+}\right), & \Lambda_{b}\left(\rho_{b}, r_{c}^{-1}\left(\rho_{+}, u_{b+}\right)\right) \tau<x<u_{b+} \tau, \\ \rho_{+}, & x>u_{b+} \tau,\end{cases}
$$

where $u_{b+}=\min \left(u_{b}, v\left(\rho_{+}\right)\right)$and $\Lambda\left(\rho_{-}, \rho_{+}\right)$is the Rankine-Hugoniot transition speed. If $v\left(\rho_{+}\right)<u_{b}$, the speed of both the platoon head and tail are set to $v\left(\rho_{+}\right)$.

- If $r_{f}\left(\rho_{b}, u_{b}\right)<\rho_{+}<r_{c}\left(\rho_{b}, u_{b}\right)$ :

$$
\rho(x, \tau)= \begin{cases}\rho_{b}, & x<W_{b} \tau, \\ \sigma_{b}, & W_{b} \tau<x<u_{b} \tau, \\ r_{f}\left(\rho_{b}, u_{b}\right), & u_{b} \tau<x<V \tau, \\ \rho_{+}, & x>V \tau .\end{cases}
$$

We have a similar situation for the Riemann problem for the moving bottleneck tail. Now, $Q_{-}(\rho)=Q_{b}(\rho)$, $Q_{+}(\rho)=Q(\rho)$ and $\rho_{+}=\rho_{b}$. Again, we have three cases of the entropy solution, depending on $\rho_{b}$ and $\rho_{+}$:

- If $\rho_{-}<r_{f}\left(\sigma_{b}, u_{b}\right)$ and $\rho_{b} \leq r_{c}^{-1}\left(\rho_{-}, u_{b}\right)$,

$$
\rho(x, \tau)= \begin{cases}\rho_{-}, & x<u_{b} \tau, \\ r_{f}^{-1}\left(\rho_{-}, u_{b}\right), & u_{b}<x<\Lambda\left(\rho_{-}, \rho_{b}\right) \tau, \\ \rho_{b}, & x>\Lambda\left(\rho_{-}, \rho_{b}\right) \tau .\end{cases}
$$




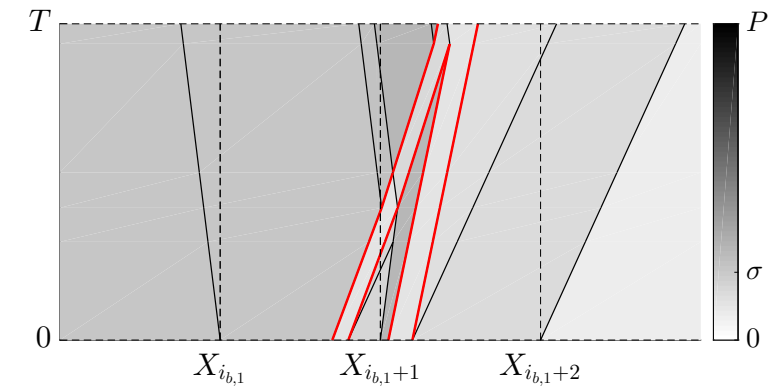

Fig. 3: The front tracking solution of two platoons merging. Platoon boundaries are shown in red.

- If $\rho_{-}>r_{f}\left(\rho_{b}, u_{b}\right)$ and $\rho_{b}>\sigma_{b}$,

$$
\rho(x, \tau)= \begin{cases}\rho_{-}, & x<\Lambda\left(\rho_{-}, r_{c}\left(\rho_{b}, u_{b}\right)\right) \tau, \\ r_{c}\left(\rho_{b}, u_{b}\right), & \Lambda\left(\rho_{-}, r_{c}\left(\rho_{b}, u_{b}\right)\right) \tau<x<u_{b} \tau, \\ \rho_{b}, & x>u_{b} \tau .\end{cases}
$$

- If $\rho_{-}>r_{f}\left(\rho_{b}, u_{b}\right)$ and $\rho_{b} \leq \sigma_{b}$,

$$
\rho(x, \tau)= \begin{cases}\rho_{b}, & x<W \tau, \\ r_{c}\left(\sigma_{b}, u_{b}\right), & W \tau<x<u_{b} \tau, \\ \sigma_{b}, & u_{b} \tau<x<V_{b} \tau, \\ \rho_{b}, & x>V_{b} \tau .\end{cases}
$$

Note that since the moving bottleneck can have length comparable to the cell length, we need to keep track of the traffic density inside its zone, in addition to keeping track of the traffic density upstream of it.

The situation when there are multiple bottlenecks in the same cell or in adjacent cells is handled by including all bottlenecks and cells in question into a single composite Riemann problem. If the tail of one and head of another bottleneck collide, we say that those two bottlenecks have merged, and take their speed to be the speed of the leader bottleneck, as shown in Fig. 3 .

To summarize, the traffic model including the moving bottlenecks we propose can now be written as

$$
\begin{aligned}
& \rho_{i}(t+1)=\rho_{i}(t)+\frac{T}{L}\left(q_{i-1}(t)-q_{i}(t)\right), \\
& q_{i}(t)=\min \left(V \rho_{i}(t), V \sigma, W\left(P-\rho_{i+1}(t)\right)+\Delta q_{i}(t),\right. \\
& {\left[\begin{array}{c}
\Delta q(t) \\
x(t+1) \\
\rho_{b-}(t+1) \\
\rho_{b}(t+1)
\end{array}\right]=\mathcal{P}\left(\rho(t), x(t), u(t), \rho_{b-}(t), \rho_{b}(t)\right),}
\end{aligned}
$$

where the map $\mathcal{P}$ encapsulate the procedure of solving composite Riemann problems for cells in the vicinity of moving bottlenecks by front tracking and calculating the updates for $q$ and new values for $x, \rho_{b-}$ and $\rho_{b}$, following the formulation given in [15].

\section{Platoon MERging in tRAFFiC}

In this section we formulate the optimization problem that we solve in order to calculate energy-optimal platoon catch-up speeds taking traffic conditions into account. First, we adopt the relative coordinate system so that the follower starts at $\chi_{2}(0)=0$, and the leader starts at $\chi_{1}(0)=\chi_{0}=x_{1}(t)-x_{2}(t)$. The goal position and time are then $\chi_{f}=x_{f}-x_{2}(t)$ and $\tau_{f}=T_{f}-t T$.

The traffic model with moving bottlenecks formulated in previous chapter can be used to predict the evolution of traffic conditions and the motion of vehicles, depending on the chosen reference speed pair $\left(u_{1}, u_{2}\right)$. However, this would lead to a very large state space, which would in turn make the optimization problem difficult. Instead, we assume that we can split the initial traffic conditions between the two vehicles into two zones. This assumption will lead to only four traffic scenarios that need to be considered, from which we will derive a scenario-dependent cost function.

Take $\rho \approx \rho_{f}, x<\chi_{c}$ and $\rho \approx \rho_{c}, x>\chi_{c}$, where $\chi_{c}$ is the minimal $x$ for which the average speed of the traffic is less than the maximum reference speed. We calculate $\rho_{f}$ and $\rho_{c}$ as average values of $\rho$ on $\left[0, \chi_{c}\right]$ and $\left[\chi_{c}, \chi_{f}\right]$ respectively. The follower vehicle can only be slowed down when it enters the zone of density $\rho_{c}$, or the zone of density $r_{c}\left(\sigma_{b}, u_{1}\right)$, originating from the leader vehicle. In further text, we denote $r_{f}\left(\sigma_{b}, u_{2}\right)$ as simply $r_{f}$ and $r_{c}\left(\sigma_{b}, u_{1}\right)$ as $r_{c}$.

From the standpoint of cost function, the four cases of traffic we need to consider are:

- Case 0: The follower is unaffected by traffic. This case was already discussed and corresponds to using cost function $J_{0}$.

- Case 1: $\tau_{\rho_{f}}<\tau_{\rho_{c}}, \tau_{\rho_{f} r_{f}}<\tau_{\rho_{f} \rho_{c}}$, the $\rho_{f}$ zone vanishes first, then $r_{f}$. The follower vehicle first runs into $\rho_{c}$, then into $r_{c}$.

- Case 2: $\tau_{\rho_{f}}<\tau_{\rho_{c}}, \tau_{\rho_{f} r_{f}}>\tau_{\rho_{f} \rho_{c}}$, the $\rho_{f}$ zone vanishes first, then $\rho_{c}$. The follower vehicle runs into $r_{c}$.

- Case 3: $t_{\rho_{f}}>\tau_{\rho_{c}}$, the $\rho_{c}$ zone vanishes first, then $\rho_{f}$. The follower vehicle runs into $r_{c}$.

The latter three cases, in which the traffic affects the vehicle speed, are shown on Fig. 4, and the front interaction times are given in Table I. We denote the transition speeds $\lambda_{\rho_{-} \rho_{+}}=\Lambda\left(\rho_{-}, \rho_{+}\right)$and the speed the follower vehicle maintains in traffic of density $\rho, u_{\rho}\left(u_{2}\right)=\min \left(u_{2}, v(\rho)\right)$. Finally, the platoon merge will occur at

$$
\begin{aligned}
\tau_{m_{i}} & =\frac{\chi_{0}-\chi_{t_{i}}}{u_{r_{c}}\left(u_{2}\right)-u_{1}} \\
\chi_{m_{i}} & =\chi_{0}+u_{1} \tau_{m_{i}}
\end{aligned}
$$

where $i=1,2,3$ is the case of traffic.

The criterion function is thus given by

$$
J(u)= \begin{cases}J_{0}(u), \quad \tau_{\rho_{f}}<\tau_{\rho_{c}},\left(\tau_{\rho_{c} \rho_{f} r_{c}}<\tau_{r_{3}} \vee \tau_{\rho_{c} r_{c}}<\tau_{\rho_{c} \rho_{f}}\right), \\ J_{1}(u), \quad \tau_{\rho_{f}}>\tau_{\rho_{c}}, \tau_{\rho_{f} r_{f}}<\tau_{\rho_{f} \rho_{c}}, \\ J_{2}(u), \quad \tau_{\rho_{f}}>\tau_{\rho_{c}}, \tau_{\rho_{f} r_{f}}>\tau_{\rho_{f} \rho_{c}}, \\ J_{3}(u), \quad \tau_{\rho_{f}}<\tau_{\rho_{c}}, \tau_{r_{3}}<\tau_{\rho_{c} \rho_{f}} r_{c}, \tau_{\rho_{c} \rho_{f}}<\tau_{\rho_{c} r_{c}},\end{cases}
$$

where

$$
\begin{gathered}
J_{1}(u)=u_{1}^{3} \tau_{m 1}+u_{2}^{3} \tau_{\rho_{f} r_{f}}+u_{\rho_{c}}^{3}\left(\tau_{r 1}-\tau_{\rho_{f} r_{f}}\right)+\ldots \\
\cdots+u_{r_{c}}^{3}\left(\tau_{m 1}-\tau_{r 1}\right)+\frac{\left(\chi_{f}-\chi_{m 1}\right)^{3}}{\left(\tau_{f}-\tau_{m 1}\right)^{2}}, \\
J_{2}(u)=u_{1}^{3} \tau_{m 2}+u_{2}^{3} \tau_{r 2}+u_{r_{c}}^{3}\left(\tau_{m 2}-\tau_{r 2}\right)+\frac{\left(\chi_{f}-\chi_{m 2}\right)^{3}}{\left(\tau_{f}-\tau_{m 2}\right)^{2}}, \\
J_{3}(u)=u_{1}^{3} \tau_{m 3}+u_{2}^{3} \tau_{r 3}+u_{r_{c}}^{3}\left(\tau_{m 3}-\tau_{r 3}\right)+\frac{\left(\chi_{f}-\chi_{m 3}\right)^{3}}{\left(\tau_{f}-\tau_{m 3}\right)^{2}} .
\end{gathered}
$$



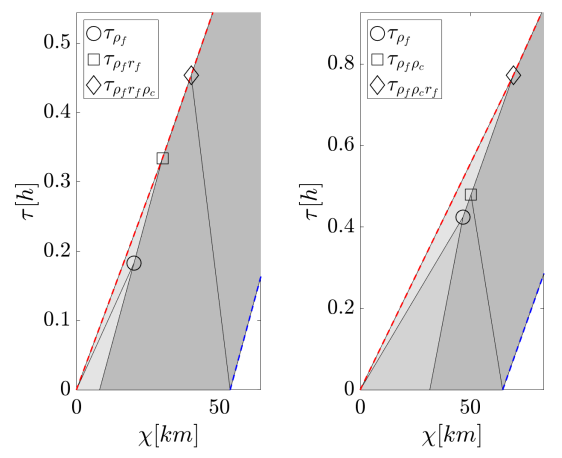

(1) $\tau_{\rho_{f}}<\tau_{\rho_{c}}, \tau_{\rho_{f} r_{f}}<\tau_{\rho_{f} \rho_{c}}$ (2) $\tau_{\rho_{f}}<\tau_{\rho_{c}}, \tau_{\rho_{f}} r_{f}>\tau_{\rho_{f} \rho_{c}}$

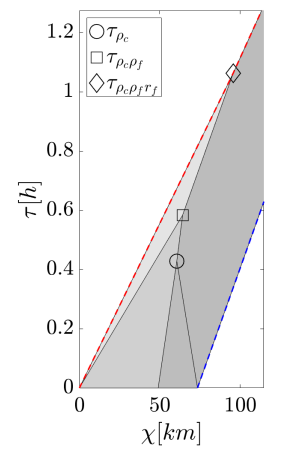

(3) $\tau_{\rho_{f}}>\tau_{\rho_{c}}$
Fig. 4: Front tracking prediction of traffic between the leader (dashed blue) and the follower (dashed red).

TABLE I: Front interaction times and positions.

\begin{tabular}{|c|c|}
\hline \multicolumn{2}{|r|}{ First } \\
\hline Case 1,2 & $\tau_{\rho_{f}}=\frac{\chi_{c}}{\lambda_{r_{f} \rho_{f}}-\lambda_{\rho_{f} \rho_{c}}}$ \\
\hline Case 0,3 & $\tau_{\rho_{c}}=\frac{x_{0}-\chi_{c}}{\lambda_{\rho_{f} \rho_{c}}+W}$ \\
\hline \multicolumn{2}{|r|}{ Second } \\
\hline Case 1 & $\tau_{\rho_{f} r_{f}}=\tau_{\rho_{f}} \frac{\lambda_{r_{f} \rho_{f}}-\lambda_{r_{f}} \rho_{c}}{u_{2}-\lambda_{r_{f}} \rho_{c}}$ \\
\hline Case 2 & $\tau_{\rho_{f} \rho_{c}}=\frac{x_{0}+\lambda_{r_{f}} \rho_{c} \tau_{\rho_{f}}-\lambda_{r_{f}} \rho_{f} \tau_{\rho_{f}}}{\lambda_{r_{f} \rho_{c}}+W}$ \\
\hline Case 3 & $\tau_{\rho_{c} \rho_{f}}=\frac{\chi_{c}-\lambda_{\rho_{f} r_{c}} \tau_{\rho_{c}}-W \tau_{\rho_{c}}}{\lambda_{r_{f} \rho_{f}}-\lambda_{\rho_{f} r_{c}}}$ \\
\hline Case $0_{1}$ & $\tau_{\rho_{c} r_{c}}=\frac{\lambda_{\rho_{f} r_{c} \tau_{\rho_{c}}-x_{0}+W \tau_{\rho_{c}}}}{\lambda_{\rho_{f} r_{c}}-u_{1}}$ \\
\hline \multicolumn{2}{|r|}{ Third } \\
\hline Case 1 & $\begin{array}{c}\tau_{t_{1}}=\tau_{\rho_{f} r_{f} \rho_{c}}=\frac{\chi_{0}+\tau_{\rho_{f}} r_{f}\left(v_{\rho_{c}}\left(u_{2}\right)-u_{2}\right)}{v_{\rho_{c}}\left(u_{2}\right)+W} \\
\chi_{t_{1}}=x_{0}-W \tau_{t_{1}}\end{array}$ \\
\hline Case 2 & $\begin{array}{c}\tau_{t_{2}}=\tau_{\rho_{f} \rho_{c} r_{f}}=\frac{\chi_{0}-\tau_{\rho_{f} \rho_{c}}\left(\lambda_{r_{f}} r_{c}+W\right)}{u_{2}-\lambda_{r_{f}} r_{c}} \\
\chi_{t_{2}}=u_{2} \tau_{t_{2}}\end{array}$ \\
\hline Case 3 & $\begin{array}{c}\tau_{t_{3}}=\tau_{\rho_{c} \rho_{f} r_{f}}=\tau_{\rho_{c} \rho_{f}} \frac{\lambda_{r_{f} \rho_{f}}-\lambda_{r_{f} r_{c}}}{u_{2}-\lambda_{r_{f}} r_{c}} \\
\chi_{t_{3}}=u_{2} \tau_{t_{3}} \\
\end{array}$ \\
\hline Case $0_{2}$ & $\tau_{\rho_{c} \rho_{f} r_{c}}=\tau_{\rho_{c}} \rho_{f} \frac{\lambda_{r_{f}} r_{c}-\lambda_{r_{f}} \rho_{f}}{\lambda r_{r_{f}} r_{c}-u_{1}}$ \\
\hline
\end{tabular}

To enforce speed and timing constraints on the vehicles, we add two additional barrier terms to $J_{i}$, corresponding to inequalities

$$
\begin{aligned}
\frac{\chi_{f}-\chi_{m_{i}}}{\tau_{f}-\tau_{m_{i}}} & \leq u_{\max }, \\
\tau_{m_{i}} & \leq \tau_{f} .
\end{aligned}
$$

Finally, the optimization problem we solve in order of finding the energy-optimal catch-up speeds for two vehicles under constraints imposed by the surrounding traffic is

$$
\begin{array}{ll}
\underset{u_{1}, u_{2}}{\operatorname{minimize}} & J(u) \\
\text { subject to } & u_{\min } \leq u_{1} \leq u_{10} \\
& u_{20} \leq u_{2} \leq u_{\max }
\end{array}
$$

This problem is not convex, but it is unimodal, so it can still be solved efficiently using numerical methods.

Having solved the optimization problem, we also obtain the optimal predicted merge time $\tau_{m_{i}}$.

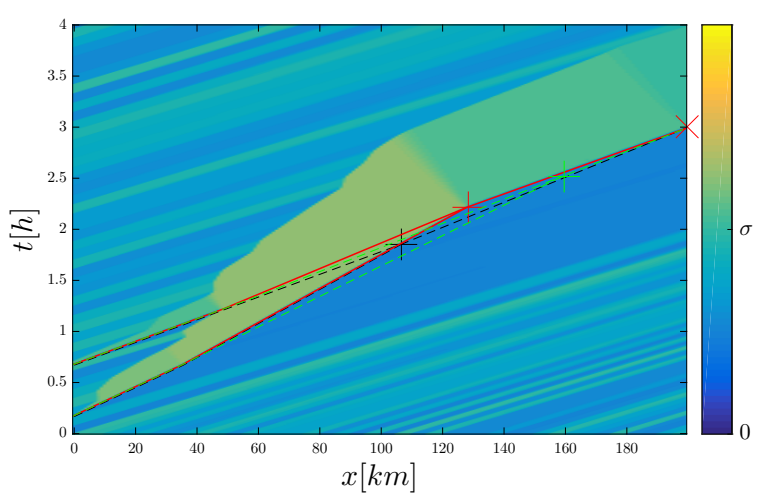

Fig. 5: An example simulation run without disturbances. Traffic density is colour-coded and crosses indicate platoon merge locations and times. Dashed black: vehicle trajectories if they were not affected by traffic. Red: vehicle trajectories using control law (2). Dashed green: vehicle trajectories if control law (3) was used.

\section{Simulation RESUlts}

We will demonstrate the effectiveness of the proposed method for calculating optimal catch-up speeds in simulations. The simulation scenario in question is as follows:

1) $t<t_{1}$ : The traffic is in free flow, with heterogeneous traffic density. The leader vehicle enters the road segment at $t=t_{1}$.

2) $t_{1} \leq t<t_{2}$ : The leader vehicle travels at speed $v_{01}$, at which it would reach $x_{f}$ at time $t_{f}$. The follower vehicle enters the road segment at $t=t_{2}$.

3) $t_{2} \leq t<t_{m}$ : The leader and the follower adjust their speeds according to the specified control law, until they merge into a platoon.

4) $t \geq t_{m}$ : The newly merged platoon proceeds and adjusts its speed so that it reaches $x_{f}$ at time $t_{f}$.

If the platoon merging fails for any of the control laws, the vehicles proceed at their maximum speed until the end of the segment, and that simulation run is not counted in average cost calculations.

The initial background traffic conditions $\rho_{i}(0)$ and inflow into the first cell $q_{0}(t)$ are randomly generated heterogeneous free flow (see Fig. 5). We used three scenarios with different traffic density ranges, light traffic $[\sigma / 5, \sigma]$, medium traffic $[\sigma / 3, \sigma]$ and heavy traffic $[\sigma / 2, \sigma]$, resulting in average traffic density of $0.6 \sigma, 0.66 \sigma$ and $0.75 \sigma$ respectively.

We are comparing three different control laws:

1) $u_{1}$ and $u_{2}$ are calculated by solving (3) once at $t=t_{2}$, ignoring traffic conditions.

2) $u_{1}$ and $u_{2}$ are calculated by solving (3) periodically during the catch-up phase, ignoring traffic conditions,

3) $u_{1}$ and $u_{2}$ are calculated by solving (4) periodically during the catch-up phase, taking traffic conditions into account.

We also considered the case when some disturbance is acting on the vehicles. Namely, at a random time instant between 5 and 10 minutes after the catch-up has begun, 


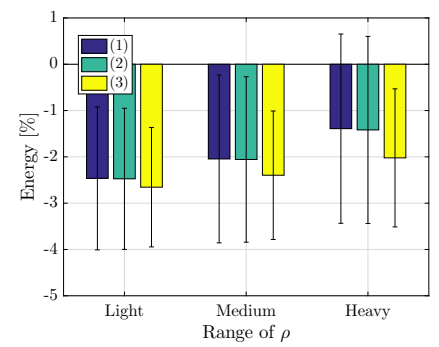

(1) No disturbances

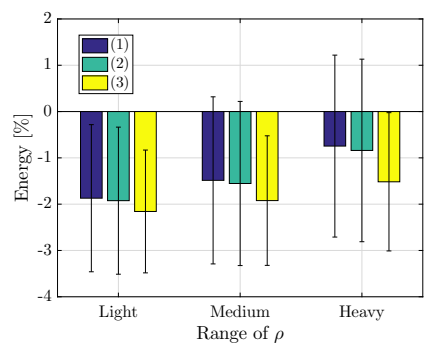

(2) With disturbance
Fig. 6: Relative energy consumption change

TABLE II: Relative energy consumption change without and with a disturbance

\begin{tabular}{|c||c|c|c||c|c|c|}
\hline \multirow{2}{*}{\multicolumn{1}{|c||}{$\rho$}} & \multicolumn{3}{c||}{ No disturbances } & \multicolumn{3}{c|}{ With disturbance } \\
\cline { 2 - 7 } & $(1)$ & $(2)$ & $(3)$ & $(1)$ & $(2)$ & $(3)$ \\
\hline Light & $-2.46 \%$ & $-2.47 \%$ & $-2.65 \%$ & $-1.87 \%$ & $-1.92 \%$ & $-2.16 \%$ \\
\hline Medium & $-2.04 \%$ & $-2.06 \%$ & $-2.40 \%$ & $-1.49 \%$ & $-1.55 \%$ & $-1.92 \%$ \\
\hline Heavy & $-1.39 \%$ & $-1.42 \%$ & $-2.02 \%$ & $-0.75 \%$ & $-0.84 \%$ & $-1.52 \%$ \\
\hline
\end{tabular}

the speed of the follower vehicle is decreased by $20 \mathrm{~km} / \mathrm{h}$ for $10 \mathrm{~min}$. This causes the platoon merging to be delayed and would result in reduced energy savings, so recalculating optimal speeds is required.

There were 100 simulation runs for each combination of control law and traffic density range, and the random seeds used to generate traffic were the same for all control laws. The average energy savings are shown in Fig. 6 and in Table II. An example simulation result is shown in Fig. 5. We can see that using control laws that take traffic conditions into consideration improves average energy savings, especially in heavy traffic. The improvement is especially apparent when disturbances are present.

In addition to calculating optimal catch-up speeds, this approach can also be used to predict when attempting to form a platoon is not beneficial. In Table III we see the number of "bad platooning attempts", i.e. in how many simulation runs the vehicles failed to reach the goal position in time, or had higher overall energy cost. If the calculated optimal catch-up speeds are such that platoon merging is predicted to occur very close to the end of the common road segment $x_{f}$, we know that attempting to form a platoon will not yield fuel savings, and may abandon the attempt at the start, instead continuing driving at vehicles' own optimal speeds. We also see the number of times the algorithm correctly predicted this outcome (true positive), which could be used to preemptively abort the platooning attempt, as well as the number of times the bad outcome was not predicted (false negative) and falsely predicted (false positive).

\section{CONCLUSION}

In this paper, we further develop the framework for introducing platoons as moving bottlenecks into the CTM. We derive a control strategy for energy-optimal catch-up of two vehicles that are attempting to form a platoon while driving on the road. It is shown in simulations that this strategy achieves better results compared to the case when we do not take traffic conditions into account. This approach has an additional benefit of being able to indicate when attempting
TABLE III: Bad platooning attempt prediction

\begin{tabular}{|c|c|c|c|}
\hline & Light & Medium & Heavy \\
\hline Total bad attempts & 5 & 15 & 23 \\
\hline Correctly predicted & 5 & 14 & 19 \\
\hline Not predicted & 0 & 1 & 4 \\
\hline Falsely predicted & 6 & 3 & 1 \\
\hline
\end{tabular}

to form a platoon would not yield improvements in fuel consumption, and thus prevent unnecessary deviations from vehicles' individual plans.

For future work, it would be interesting to also investigate other platooning maneouvres, such as reordering the vehicles, in a similar framework. Additionally, the control strategy is derived for a macroscopic traffic model, and it would be beneficial to test it in microscopic simulations.

\section{REFERENCES}

[1] A. Alam, B. Besselink, V. Turri, J. Martensson, and K. H. Johansson, "Heavy-duty vehicle platooning for sustainable freight transportation: A cooperative method to enhance safety and efficiency," IEEE Control Systems, vol. 35, no. 6, pp. 34-56, 2015.

[2] C. Bergenhem, S. Shladover, E. Coelingh, C. Englund, and S. Tsugawa, "Overview of platooning systems," in Proceedings of the 19th ITS World Congress, Vienna, Austria, 2012.

[3] H. L. Humphreys, J. Batterson, D. Bevly, and R. Schubert, "An evaluation of the fuel economy benefits of a driver assistive truck platooning prototype using simulation," SAE Technical Paper, Tech. Rep., 2016.

[4] R. Horowitz and P. Varaiya, "Control design of an automated highway system," Proceedings of the IEEE, vol. 88, no. 7, pp. 913-925, 2000.

[5] V. Turri, B. Besselink, J. Mårtensson, and K. H. Johansson, "Fuelefficient heavy-duty vehicle platooning by look-ahead control," in 53rd IEEE Conference on Decision and Control. Los Angeles, US: IEEE, 2014, pp. 654-660.

[6] A. K. Bhoopalam, N. Agatz, and R. Zuidwijk, "Planning of truck platoons: A literature review and directions for future research," Transportation Research Part B: Methodological, 2017.

[7] S. van de Hoef, K. H. Johansson, and D. V. Dimarogonas, "Fuelefficient en route formation of truck platoons," IEEE Transactions on Intelligent Transportation Systems, vol. 19, no. 1, pp. 102-112, 2018.

[8] K.-Y. Liang, J. Mårtensson, and K. H. Johansson, "When is it fuel efficient for a heavy duty vehicle to catch up with a platoon?" IFAC Proceedings Volumes, vol. 46, no. 21, pp. 738-743, 2013.

[9] —-, "Experiments on platoon formation of heavy trucks in traffic," in 19th IEEE International Conference on Intelligent Transportation Systems (ITSC), Nov 2016, pp. 1813-1819.

[10] M. Saeednia and M. Menendez, "Analysis of strategies for truck platooning: Hybrid strategy," Transportation Research Record: Journal of the Transportation Research Board, no. 2547, pp. 41-48, 2016.

[11] W. Zhang, E. Jenelius, and X. Ma, "Freight transport platoon coordination and departure time scheduling under travel time uncertainty," Transportation Research Part E: Logistics and Transportation Review, vol. 98, pp. 1-23, 2017.

[12] K.-Y. Liang, Q. Deng, J. Mårtensson, X. Ma, and K. H. Johansson, "The influence of traffic on heavy-duty vehicle platoon formation," in IEEE Intelligent Vehicles Symposium (IV). Seoul, Korea: IEEE, 2015, pp. 150-155.

[13] M. Čičić, K.-Y. Liang, and K. H. Johansson, "Platoon merging distance prediction using a neural network vehicle speed model," IFAC-PapersOnLine, vol. 50, no. 1, pp. 3720-3725, 2017.

[14] C. F. Daganzo, "The cell transmission model: A dynamic representation of highway traffic consistent with the hydrodynamic theory," Transportation Research Part B: Methodological, vol. 28, no. 4, pp. 269-287, 1994.

[15] M. Čičić and K. H. Johansson, "Traffic regulation via individually controlled automated vehicles: a cell transmission model approach," in 21st International IEEE Conference on Intelligent Transportation Systems (ITSC), Maui, US, 2018.

[16] M. L. Delle Monache and P. Goatin, "Scalar conservation laws with moving constraints arising in traffic flow modeling: an existence result," Journal of Differential equations, vol. 257, no. 11, pp. 40154029, 2014. 